\title{
Methods for achieving enhanced temporal resolution in IR image acquisition
}

\author{
by S.M. SHEPARD, D.T. SASS, T.P. IMIROWICZ
}

Applied Research Branch (AMSTA-RSA), U.S. Army Tank-Automotive Command

Warren, Michigan 48397-5000, USA

\begin{abstract}
Temporal aliasing is a problem common to all video imaging systems. In the infrared, targets which undergo temperature variations at frequencies above the frame rate will be inaccurately imaged. We have developed two novel methods for achieving Enhanced Temporal Resolution (ETR), using optomechanical and focal plane array technology. These systems employ phase synchronous detection. methods to compensate for the effects of aliasing, and allow above-frame-rate events to be imaged. The optomechanically based ETR system utilizes an unmodified 8-12 $\mu \mathrm{m}$ commercial imager (Inframetrics IR 600 ), and allows images of repetitive thermal events at frequencies up to $4 \mathrm{kHz}$ to be imaged with an effective integration time of $125 \mu \mathrm{s}$. Data is acquired selectively by comparing the motion of the camera's horizontal scanning mirror to a reference signal correlated to the event of interest. The focal plane array ETR system is based on a $160 \times 244 \mathrm{PtSi}$ Schottky-barrier sensor operating at the standard $30 \mathrm{~Hz}$ frame rate. Frame integration time is variable to a minimum of $10 \mu \mathrm{s}$. Data is acquired synchronously with respect to an external reference signal derived from the target. The relative merits and weaknesses of the two approaches are discussed.
\end{abstract}

\section{Introduction}

Current research in IR imaging has focused primarily on improving spatial resolution and radiometric accuracy of detectors, while far less attention has been paid to the temporal content of the IR video image. For scanning systems, each displayed pixel represents an RMS average of the scene irradiance integrated over the dwell time, and acquired at a different time than every other pixel in the field. For this type of system, the interval between the first and last acquired pixels can be taken as the effective integration time of the imaging system. For RS170 compatible units this time is typically $33 \mathrm{~ms}$ which is too long to resolve many transient thermal events of interest in NDE applications.

For focal plane array (FPA) type imagers, many interline transfer designs capture all pixels in a video field simultaneously, although they are read out sequentially. Unfortunately, the integration time is generally set to be the inverse of the frame rate, resulting in the same $33 \mathrm{~ms}$ integration time found in optomechanical systems.

The effect of a long integration time when viewing high speed events is seen as blurring of the image, while the low frame rate results in aliasing error when repetitive events are imaged. Compared to visible images, the presence of aliasing error can be difficult to detect in IR images. This is due to their typically low contrast, and the fact that many IR targets of interest are spatially stationary, but thermally time-varying. Objects in motion suffer geometrical distortion due to aliasing, which is easily identified by comparing target and image geometries, while stationary IR images undergo thermal distortion when aliased, a much more subtle effect. 


\section{http://dx.doi.org/10.21611/qirt.1992.002}

We have developed two systems to address the problems associated with the fixed frame rate and integration time of RS-170 optomechanical and focal plane array imagers [1-5]. Both methods employ synchronous data acquisition, in order to detect the phase (or equivalently the temporal relationship) of each acquired pixel with respect to some time varying feature of the target. In this sense, both methods can be regarded as video boxcar averaging techniques. However, the acquisition schemes used in the two systems are very different, and achieve optimum performance for different types of targets. In both systems, we have avoided solutions involving non standard frame rates, in order to avoid the additional problems associated with high speed data communication and storage. Instead, we have opted to retain the $30 \mathrm{~Hz}$ RS-170 frame rate, so that commercial frame grabber and image processing boards could be employed.

\section{Enhanced temporal resolution imaging}

\subsection{Line-by-line method}

Aliasing errors are the result of undersampling, and the fact that input to the imager is not band limited. For a conventional imaging system, the sampling frequency is equivalent to the frame rate $(30 \mathrm{~Hz})$. In our approach, only horizontal scan lines with a prescribed phase relationship to the target temporal variation are acquired. The image is built from lines extracted from a number of successive fields, and each horizontal line has the same phase, with respect to the target reference signal, as every other line in the image. The effective sampling frequency of the system is the horizontal scan frequency, which is typically two orders of magnitude higher than the frame rate.

The imager (Inframetrics IR-600) is a commercial 8-12 $\mu \mathrm{m}$ optomechanical scanning unit. Its horizontal scanning mirror sweeps the object field in a bidirectional, sinusoidal pattern at 4 $\mathrm{kHz}$. Data is acquired during both forward (left to right) and reverse (right to left) passes of the mirror.

The image acquisition process is shown in greater detail in figure 1, which shows three consecutive horizontal scan lines. The synchronizer hardware generates an aperture which has a programmable width $(\Delta t)$ and offset from the beginning of the line $(\Delta \varnothing)$. When the leading edge of the reference signal associated with the target falls within the aperture, a signal is sent to the host microcomputer to freeze the frame and save the good line (in this case line $n$ ) to a memory buffer where it is averaged with previous acquisitions of the same line and then displayed. In the resulting image, all horizontal lines have the same phase with respect to the reference signal, and the effective integration time is equivalent to the time required to scan a single horizontal line (125 $\mu$ s for the Inframetrics IR-600).

Images are built from horizontal lines extracted from many successive video fields. Several good lines may be extracted from a single video field (the current system allows three lines to be extracted per field). Each time more good lines are added to the RAM buffer, the display is updated, and the evolving image is displayed on screen, along with a histogram which represents the number of times each line has been sampled. Image acquisition time is a function of the target frequency and $\Delta t$, the width of the aperture. Data acquisition is controlled by an IBM compatible 80386 micro-computer, equipped with a frame grabber board (Thermoteknix GRAM 1) and an RGB monitor. The software for this system was developed in house, and contains a number of features specially developed for the line-by-line acquisition mode. The user may specify the minimum and maximum number of times horizontal lines are to be acquired and averaged, so that the final image consists of lines which have all been averaged the same number of times. Another feature allows images to be acquired in a 
subtractive mode, where time resolved images taken at two different time offsets are acquired during the same acquisition cycle, and then subtracted. The resulting image displays only those features of the scene which vary synchronously with the reference signal.

\subsection{Focal plane array method}

The obvious solution to the problems of aliasing and blurring with a focal plane imager would be to increase the frame rate of the system. However, IR focal plane arrays capable of imaging at the effective frame rate of the line-by-line system $(4 \mathrm{kHz})$, are not currently available. Instead, we opted to reduce the actual integration time of the camera, and utilize synchronous data acquisition to limit the amount of acquired data to only those fields with a prescribed phase with respect to the target reference. The system uses an off the shelf $320 \mathrm{x}$ 244 PtSi Schottky-barrier CCD image sensor from David Sarnoff Laboratories, which operates in the $3-5 \mu \mathrm{m}$ range. The PtSi sensor is mounted into a liquid nitrogen pour-fill dewar. IR radiation is focused on the PtSi sensor by means of an $f / 1.6$ antireflection coated $36 \mathrm{~mm} \mathrm{Ge} / \mathrm{Si}$ doublet lens.

Control signals to the sensor are sent to an electronic controller, which allows the user to control voltage levels of the PtSi chip, integration time, and mode of operation (normal, internal trigger, external trigger). In addition, the controller houses a correlated double sampling (CDS)/video mixer board. The camera system operates asynchronously in normal mode at 30 frames per second. Each frame has $320 \times 244$ pixels with two vertically interlaced $320 \times 122$ element fields per frame. The pixels are represented by potential well charge packages. To transfer charge packets, a positive pulse is applied directly opposite the detectors of a given field in the CCD.

Once the charge has been transferred to the vertical register, it is then clocked downward, using the four phase B clocks, in parallel to a horizontal multiplexer. Charge is sent out serially from the horizontal multiplexer to the support electronics. The resulting composite video signal contains active video at every other line.

In the short integration time mode the operation of the $B$ clocks is modified to a transferdump-transfer sequence. A short $(0.77 \mu \mathrm{s})$ transfer pulse, corresponding to the synchronizing trigger from the target, transfers charge accumulated since the last transfer from the detector to the vertical shift registers. All vertical clock voltages are then lowered, allowing the charge to diffuse into the substrate. A second transfer pulse is then applied, and the charge packets are read out in the normal manner. The time between the two transfer pulses is the integration time, which can be adjusted to a minimum of $10 \mu \mathrm{s}$.

\section{Comparison of line-by-line and focal plane methods}

The different spectral ranges of the two systems, and the instantaneous versus extended. image acquisition times of the respective focal plane and line-by line systems make each of the systems appropriate for different types of applications. The line-by-line technique is best suited to acquire images without aliasing error in situations where the target is undergoing a periodic temperature change at above-frame-rate frequencies. It is also useful in low frequency $(<30 \mathrm{~Hz})$ cases when the phenomena of interest is a fast transient on a time scale much shorter than the frame integration time, e.g. the propagation of the flame in an internal combustion engine. Advantages of this method are the high degree of temporal coherence, absence of aliasing up to $4 \mathrm{kHz}$, and the short effective integration time. 


\section{http://dx.doi.org/10.21611/qirt.1992.002}

It should be noted that the improvement in effective integration time that this technique affords is at the expense of the acquisition time, which is proportional to both the frequency of the target, and the precision of the timing aperture. For a high frequency target $(>2 \mathrm{kHz}) 90 \%$ of the horizontal lines are acquired within a few seconds. However, acquiring a complete image with uniform image statistics (e.g. acquire exactly five repetitions of every horizontal line, and display the linear average of the repititions) may take 2-4 min. A below-frame-rate target would have an even longer acquisition time. As an example, a $20 \mathrm{~Hz}$ target typically requires $7 \mathrm{~min}$ to generate a complete image. Images acquired this way show excellent contrast and signal-to-noise improvement due to the high degree of temporal coherence and the averaging of subsequent captures of a given line. However, many targets are not sufficiently repetitive, or stable over the image acquisition time, to be imaged with this technique.

The reduced integration time PtSi system has a nearly instantaneous acquisition time, as only a single frame must be captured to generate a complete image. However, the synchronous data acquisition scheme employed dictates that an image will not be captured until the prescribed coincidence occurs between the reference signal from the target and the extra clock cycle. For even slightly above-frame-rate events this coincidence occurs frequently enough that only a slight flicker is perceived, and diminishes as the target frequency is increased.

A more serious problem in the PtSi system is the reduced photon flux which occurs as a result of the shortened integration time. The PtSi camera is sensitive to radiation in the $2-5 \mu \mathrm{m}$ band, and is therefore better suited to look at hotter targets. As the integration time is reduced so is contrast, so that at very short integration times only scenes with high target/background contrast will produce satisfactory images. This situation can be remedied somewhat by integrating over several frames, but in general the $\mathrm{HgCdTe}$ based line-by-line system offers superior results when imaging room temperature targets.

\section{Conclusion}

At present, the basic tradeoff between the line-by-line and focal plane systems is acquisition time versus image quality and radiometric accuracy. For high-frequency, stable events, the line-by-line system produces images free of aliasing error, with higher spatial, temporal, and radiometric resolution than the same imager used in the conventional manner. The $8-12 \mu \mathrm{m}$ passband of the HgCdTe imager allows room temperature objects to be imaged with a high degree of contrast.

The PtSi based method is not appropriate for room temperature targets. The principal advantage of this system is the fast acquisition time it offers. It can be used for either repetitive or single shot events. The system has not been radiometrically calibrated, and is not yet appropriate for quantitative analysis.

\section{Acknowledgements}

The authors gratefully acknowledge Prof. Eustace Dereniak and Laurence Flath of the University of Arizona Optical Sciences Center, and John Garcia, of Hughes Aircraft, for their work in the development of the short integration time PtSi focal plane array camera. 


\section{REFERENCES}

[1] SHEPARD (S.M.), SASS (D.T.) and IMIROWICZ (T.). - Time-resolved video-thermography at above-frame-rate frequencies, SPIE (1467) Thermosense XIII, 1991, p. 234.

[2] SHEPARD (S.M.) and D.T. SASS. - Thermal imaging at above-frame-rate frequencies, Optical Engineering, (29) 2, Feb. 1990, p. 105.

[3] SHEPARD (S.M.), SASS (D.T.) and IMIROWICZ (T.). - Enhanced temporal resolution with a scanning imaging radiometer, Optical Engineering, (30) 11, Nov. 1991, p. 1716.

[4] GARCIA (J.P.), DERENIAK (E.L.) and SHEPARD (S.M.). - High speed infrared camera, Reviews of Scientific Instrumentation, (accepted for publication, 1992).

[5] FLATH (L.), DERENIAK (E.L) and SHEPARD (S.M.). - Development of a high speed PtSi IRCCD camera, SPIE Proceedings, IR Detectors and Focal Plane Arrays, Vol. 1685, to be published, 1992.

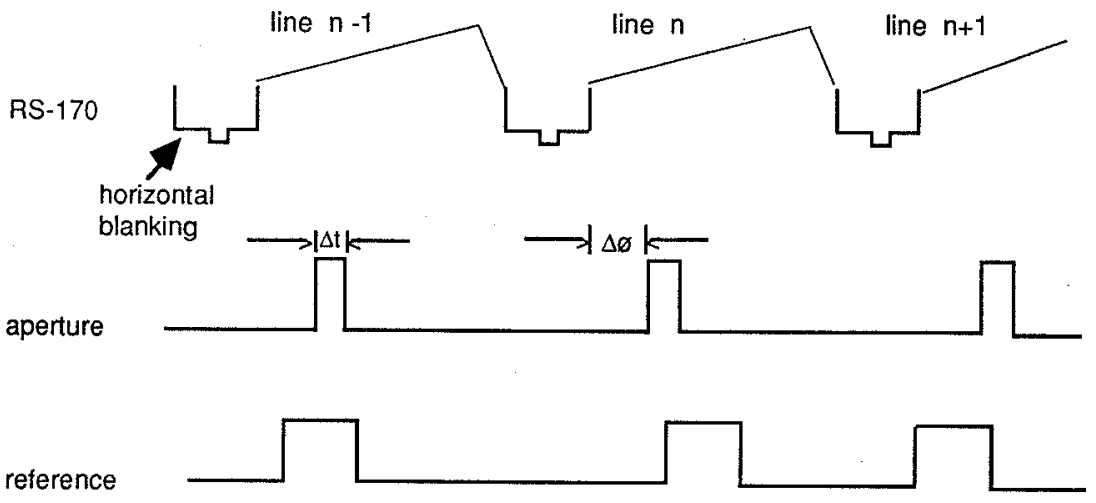

Fig. 1 - Timing of the line-by-line method. Line $n$ is a good video line. 